\title{
DESENVOLVIMENTO DE PROTÓTIPO DE RTO
}

\author{
M. EBRAHIMPOUR ${ }^{1}$, J. O. A. MATIAS ${ }^{1}$, J. E.A. GRACIANO ${ }^{1}$, E. E. MARTÍNEZ ${ }^{1}$, A. M. A. \\ PEÑA ${ }^{1}$, F.D. RINCON ${ }^{1}$, D.F. MENDOZA ${ }^{4}$, A. D. QUELHAS ${ }^{2}$, R. DE P. SOARES ${ }^{3}$, F. LIPORACE ${ }^{2}$, \\ D. ODLOAK ${ }^{1}$, A. S. VIANNA. JR ${ }^{1}$, G. A. LE ROUX ${ }^{1}$ \\ ${ }^{1}$ USP, Escola Politécnica, Departamento de Engenharia Química \\ ${ }^{2}$ Petrobras \\ ${ }^{3}$ Universidade Federal do Rio Grande do Sul \\ ${ }^{4}$ Universidad Autónoma del Caribe, Departamento de Ingenieria Mecánica \\ E-mail para contato: galoroux@usp.br
}

\begin{abstract}
RESUMO - A técnica de Otimização em Tempo Real (RTO) visa levar um processo às condições ótimas de operação mesmo em um cenário sujeito a perturbações e a limitações. Os algoritmos de RTO contemplam: a detecção de estados estacionários, a detecção de erros grosseiros, a reconciliação de dados, a estimação de parâmetros, otimização do processo, a análise econômica e deve ser compatível com a estrutura de controle de processos. No cerne de tudo isto está um modelo de processo. Para a modelagem foi adotada a plataforma EMSO porque é orientada a equações, orientada a objeto, além de ter sido desenvolvida no Brasil por universidades e empresas, com a participação da Petrobras. Neste trabalho é apresentada a arquitetura de um protótipo que permite que sejam testados diferentes algoritmos para as diferentes etapas do RTO que será aplicada em uma unidade de separação da Petrobras. São estudadas e comparadas metodologias de detecção de estados estacionários, de estimação de parâmetros e de otimização.
\end{abstract}

\section{INTRODUÇÃO}

A crescente competitividade no setor de petróleo e derivados no Brasil e no mundo associada a custos operacionais crescentes do Refino e especificações de produto progressivamente mais restritas impõem a necessidade de adoção de novas tecnologias de otimização da operação dos processos de refino. A otimização em tempo real (RTO) define periodicamente o ponto ótimo de operação e o envia automaticamente para o controle avançado (on-line). O processo opera continuamente neste ponto ótimo, com confiabilidade e baixa variabilidade.

O método clássico e mais natural de projetar a camada de RTO é otimizar uma função objetivo econômica sujeita a um modelo fenomenológico em estado estacionário que descreve o comportamento do processo. Essa estratégia ganhou destaque no final dos anos 1980, quando algumas circunstâncias permitiram a aplicação deste tipo de RTO, a saber: modelagem orientada à equação, capacidade do processamento dos computadores e desenvolvimento de novos solvers de otimização 
de grande porte, (DARBY et al., 2011).

A estrutura clássica do algoritmo de RTO é apresentada na Figura 1. Inicialmente, as medições do processo real são analisadas por um procedimento de detecção de estado estacionário que ativa o ciclo de RTO. Então, o ponto estacionário é filtrado pelos procedimentos de detecção de erros grosseiros e reconciliação de dados. Essa informação é usada na seção de estimação de parâmetros onde o modelo é atualizado, e por fim, o modelo é otimizado obtendo-se o valor ótimo das variáveis manipuladas, os quais alimentam a camada de controle de processo.

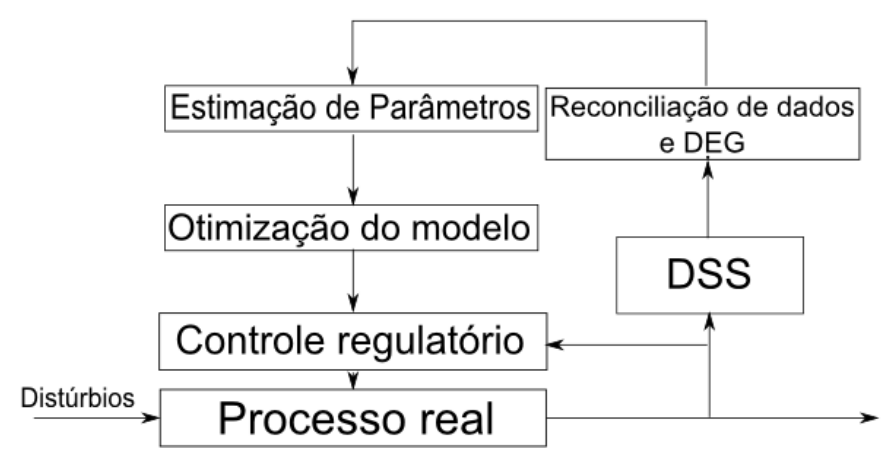

Figura 1: Estrutura clássica do RTO

A Petrobras tem investido na avaliação da tecnologia RTO através de implantações piloto em unidades industriais, utilizando soluções comerciais. Tais soluções são de fácil utilização e estão em contínua evolução, mas, frequentemente, as empresas que as produzem não possuem modelos para os processos de conversão usuais na indústria do refino. Nos casos em que tais modelos estão disponíveis há incertezas em relação à sua qualidade pelo fato de que as empresas não são refinadoras e não possuem acesso a um grande banco de dados operacionais para aperfeiçoá-los continuamente. Para fazer frente a essa situação, as maiores empresas de petróleo do mundo, como por exemplo, a Exxon-Mobil, utilizam uma abordagem híbrida, incorporando modelos de processos de desenvolvidos internamente aos ambientes comerciais de modelagem.

Outra questão importante a ser levantada é que as aplicações de RTO realizadas pela Petrobras até o momento mostram que a tecnologia empregada não é totalmente transparente. Dentro deste cenário, o projeto visa o desenvolvimento de um protótipo para implementação do RTO em uma unidade industrial da Petrobras e ganhos incrementais em relação ao estado-da-arte atual. Dentre as principais características desse protótipo, destaca-se a utilização da plataforma de modelagem EMSO como motor de cálculo, porque é orientada a equações, orientada a objeto, além de ter sido desenvolvida no Brasil por universidades e empresas, com a participação da Petrobras, além disso, o desenvolvimento do protótipo de RTO considera uma arquitetura orientada objetos de maneira a torná-lo suficientemente flexível para implementar as diversas metodologias disponíveis para cada etapa do processo. 


\section{9 a 22 de outubro de 2014 \\ Florianópolis/SC}

O presente trabalho está dividido em sete capítulos onde serão descritos os principais problemas observados, possíveis soluções e trabalhos desenvolvidos na área, além de produção bibliográfica do presente grupo de pesquisa. $\mathrm{O}$ capitulo dois trata do problema da detecção do estado estacionário, $\mathrm{o}$ capitulo três da estimação de parâmetros, o problema da otimização dentro do RTO é discutido no capitulo quatro, o quinto capítulo fala sobre a arquitetura desenvolvida para o protótipo, o caso de estudo a ser implementado na indústria é apresentado no capítulo seis, por fim, algumas discussões e conclusões são expostas no capítulo sete.

\section{DETECÇÃO DE ESTADOS ESTACIONÁRIOS}

O RTO depende crucialmente de metodologias de estimação de estado estacionário (SSI, sigla normalmente utilizada em inglês para esta técnica) porque o esquema clássico de RTO usa um modelo estacionário para os seus cálculos, este modelo representa diversas condições de operação mediante a atualização de seus parâmetros. Além disso, SSI possui dois graus de dificuldade: o processamento de dados ruidosos e grosseiros e implementação dos métodos de SSI (KORBEL et al., 2014), os quais têm atraído muita atenção em várias investigações.

As análises de estado estacionário têm sido realizada com diferentes estratégias na literatura. Por exemplo, CAO e RHINEHART,(1995) propuseram uma metodologia baseada na comparação de duas variâncias para a determinação do estado estacionário. LE ROUX et al., (2008) e TAO et al., (2012) propuseram uma técnicas baseadas em equações polinomiais, considerando um tamanho fixo de uma janela móvel de dados, no qual a determinação de SSI foi definida mediante o uso da inclinação do polinômio como índice ou valor crítico. No presente projeto de RTO estão sendo exploradas as técnicas de CAO e RHINEHART, (1995), TAO et al., (2012) e nossa proposta que considera a construção de um modelo ARX, sendo que a singularidade das matrizes do modelo ARX são usadas como índice para a determinação do estado estacionário.

\section{ESTIMAÇÃO DE PARÂMETROS}

Teoricamente, enquanto um modelo torna-se matematicamente mais complexo e mais fenomenológico, permite potencialmente uma representação mais ampla do comportamento do sistema. No entanto, a principal desvantagem associada a modelos complexos é a quantidade de informação experimental requerida sobre os mecanismos internos, que são frequentemente contaminados pelo ruído das medidas disponíveis, ampliando possíveis fontes de incertezas. Este é o cenário em que o RTO se encontra (modelos complexo, pouca informação disponível e com ruído), tornando comuns os problemas identificabilidade do modelo, e posteriormente problemas de predição.

A preocupação com essa etapa limitante do ciclo de RTO levou ao estudo de quatro técnicas de estimação de parâmetros, com intuito de selecionar dentre elas a mais promissora para ser introduzida no protótipo. Foram consideradas duas técnicas baseadas na sensibilidade do problema com relação aos parâmetros e análise de autovalores (Discriminação Rotacional e Seleção automática e Parâmetro), reparametrização via geometria diferencial e o método clássico dos mínimos quadrados não lineares. Os métodos foram avaliados em termos de capacidade de predição no conjunto de 
validação, robustez e velocidade. A metodologia de Monte Carlo foi adotada para avaliar a informação estatística necessária para quantificar a incerteza inerente a cada abordagem.

Os resultados mostram que o método de Discriminação Rotacional apresenta as melhores características de entre os métodos avaliados, uma vez que requer menos informação a priori que o método de re-parametrização por geometria diferencial, utiliza critérios de parada mais simples que o método de Seleção Automática de Parâmetros, e reduz o sobreajuste causado pelo método dos mínimos quadrados não lineares, além de estimar conjuntos de parâmetros com maior capacidade de predição dentre os métodos testados. Os resultados obtidos nesse trabalho foram publicados em revista internacional e podem ser analisados com mais detalhes em GRACIANO et al, (2014).

\section{OTIMIZAÇÃO}

A otimização econômica em um sistema RTO consiste em determinar o ponto operacional que melhor satisfaça o critério de desempenho econômico do processo produtivo estudado. $\mathrm{O}$ desempenho econômico é expresso matematicamente através de um problema de otimização com uma função objetivo que pode representar, por exemplo, o lucro bruto operacional e, o espaço de busca do ponto ótimo é limitado normalmente por restrições operacionais, capacidade produtiva e especificações de pureza dos produtos entre outros.

Considerando-se que o problema de otimização engloba toda a refinaria, uma única unidade otimizada cobre apenas um subconjunto do problema e como tal, não necessariamente fornece uma resposta válida. Ainda assim, há um argumento a favor das otimizações locais das unidades de produção, este argumento é que a modelagem de uma refinaria completa é muito complexa de ser feita, então o melhor a se fazer é tirar proveito das otimizações locais. Só existe uma maneira de executar isso corretamente, especificando corretamente os preços dos produtos das unidades da refinaria. Uma vez que os preços dos produtos são conhecidos, a otimização individual de cada unidade pode maximizar o lucro da refinaria como um todo. No entanto, os produtos das unidades não são sempre produtos finais das refinarias, e faz-se necessário especificar os preços dos produtos intermediários, que são função da qualidade, do fluxo e também de considerações na programação da produção, (FRIEDMAN, 1995).

O principal critério para atribuir um preço a um produto intermediário é a existência de preço de mercado para esse produto, porém, se não existisse o preço de mercado para determinado produto este pode ser estimado a partir de programação matemática, estes preços são denominados preços sombra. Os preços sombra e preços de mercado são os dois principais recursos para estimar-se os preços das correntes intermediários.

Resolver problemas de otimização de modelos de processos de separação, típico da indústria petroquímica, pode resultar num problema com elevado número de restrições e variáveis, o que pode ocasionar inviabilidade computacional devido ao elevado número de restrições e variáveis ou simplesmente pelo tempo requerido para ser resolvido, comprometendo a utilidade do modelo. É por isso que é importante estudar técnicas de decomposição como relaxação Lagrangeana e Lagrangeano aumentado entre outros. Estes técnicas baseiam-se em algoritmos iterativos para que os problemas de 


\section{9 a 22 de outubro de 2014 \\ Florianópolis/SC}

otimização possam ser decompostos e resolvidos separadamente, realizando atualizações do multiplicador de Lagrange para as restrições complicadoras, onde para fins económicos os multiplicadores de Lagrange representariam os preços sombra dos produtos intermediários. Essas técnicas de decomposição vêm sendo estudadas por nosso grupo de pesquisa com intuito de aplica-las no protótipo.

Outro problema relacionado com a otimização no ciclo de RTO é a presença de desvios entre as predições do modelo e a planta real. Por esse motivo, apesar da etapa de estimação de parâmetros, o modelo não é capaz de encontrar o ponto ótimo operacional do processo real, resultando numa operação sub-ótima da planta.

Algumas metodologias foram desenvolvidas para lidar com esse problema. O primeiro método foi proposto por ROBERTS, (1979) e é uma modificação do método de clássico de RTO, neste caso o problema de estimação dos parâmetros e de otimização são integrados, resultando numa função objetivo econômica modificada, tornando o ISOPE (Integrated System Optimization and Parameter estimation) capaz de lidar com o problema de incompatibilidade estrutural entre modelo e planta. O segundo método é o MA (Modifier Adaptation) proposto por MARCHETTI et al, (2009), a principal diferença desse com relação ao método clássico é a forma com que a informação da planta é utilizada, já que as medições são utilizadas para satisfazer as condições necessárias de otimalidade da planta (por meio dos chamados modificadores). O último método de RTO criado para lidar com a incompatibilidade estrutural entre modelo e planta é o algoritmo SCFO (Sufficient Conditions for Feasibility and Optimality), desenvolvido por BUNIN et al, (2013), tem como ideia principal, combinar os conceitos de direção de descenso e limite superior quadrático para obter condições suficientes que garantam um custo decrescente em cada iteração, e também, os conceitos de restrições aproximadamente ativas e continuidade de Lipschtz para garantir a viabilidade das restrições em cada passo.

O grande desafio impostos por esses três métodos alternativos é a necessidade de avaliar as derivadas das saídas do processo real com relação às entradas, o que na prática, é um processo custoso e impreciso. Por esse motivo, faz-se necessária a comparação destes com o método clássico num mesmo cenário para avaliação das vantagens e desvantagens de cada algoritmo. Um primeiro estudo foi publicado em congresso internacional GRACIANO et al, (2013) utilizando a metodologia de Monte Carlo para a avaliação das incertezas estudadas.

\section{PROTÓTIPO}

Devido à diversidade de alternativas disponíveis para se lidar com cada problema dentro do ciclo RTO, faz-se necessária a criação de um protótipo flexível que permita mudança no fluxo de informação e das ferramentas utilizadas. O software framework é uma abstração em que funcionalidades genéricas podem ser substituídas seletivamente, a fim de desenvolver aplicações de domínio específico. Ele fornece uma arquitetura em blocos de construção para uma família de sistemas e também lugares onde adaptações devem ser feitas (BUSCHMANN et al., 1996). O comportamento padrão do framework garante cumprir sua missão de uma forma pré-definida. Em ambientes orientados a objeto este comportamento pode ser modificado, simplesmente, pela 
substituição de classes abstratas e interfaces primordiais.

A Figura 2 mostra a interação do protótipo com os sistemas de computadores da planta recebendo dados de processo reais e enviando soluções RTO calculadas. O bloco de Processo RTO é a estrutura que contém os componentes responsáveis: pelo fluxo de informações (schedules), pela requisição de informação da planta, execução de cálculos (que utiliza o software EMSO como plataforma de modelagem e solução de problemas numéricos) e analise dos resultados obtidos. Mais detalhes podem ser encontrados no trabalho publicado no congresso ESCAPE 24, Matínes et al., (20140).

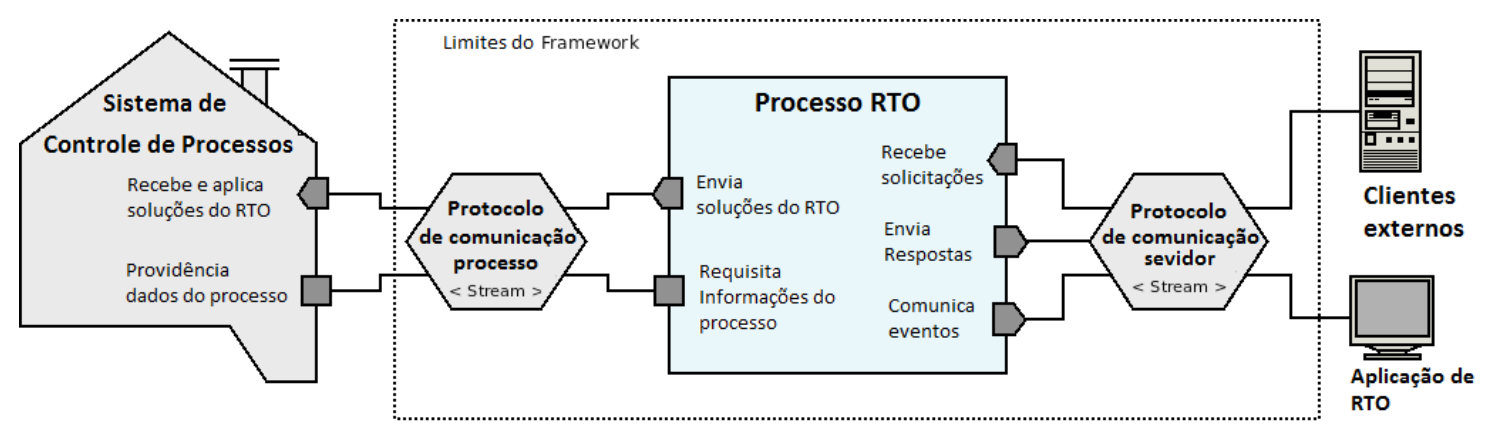

Figura 2: Representação do nível zero da arquitetura do protótipo RTO

Este trabalho pode abrir novos horizontes para aplicações RTO mais sofisticadas, dando chances de estudar na prática combinações inexploradas de técnicas. Formando uma bancada para a exploração questões envolvidas não apenas no RTO, como também, em outras alternativas disponíveis na literatura para a otimização de processos. Já que está sendo desenvolvida de maneira flexível e possibilita a comunicação com ferramentas disponíveis em unidades de produção reais.

\section{CASO DE ESTUDO}

Pretende-se aplicar o RTO em uma unidade de separação de propeno da Petrobras, mais especificamente na sua coluna depropenizadora. A escolha foi motivada pelo grande consumo energético e pela alta razão de refluxo do processo, características intrínsecas de processos de separação envolvendo misturas com pontos de ebulição próximos, que os tornam um tema atrativo para pesquisas de otimização visando melhoras no rendimento energético, assim como na economia de energia na unidade, (LÜ et al., 2010).

A configuração típica de um sistema de destilação com recompressão de vapor é similar aos demais sistemas de destilação, exceto pela utilização de parte do vapor de topo da coluna como fonte de calor para o seu refervedor. O vapor é comprimido em um compressor para facilitar a transferência de calor para as correntes de fundo da torre. Além do refervedor, um trocador de calor auxiliar é usado para condensar o vapor residual, desta maneira, controlando a pressão da torre, assim como a temperatura do refluxo, (JOGWAR e DAOUTIDIS, 2009). Esse sistema introduz uma interação forte entre as diferentes unidades no sistema. 


\section{9 a 22 de outubro de 2014 \\ Florianópolis/SC}

Como modelos mecanísticos e ambientes de simulação orientada a objetos (EO) são mais adequados para aplicações em RTO, (MEIXELL et al, 2010). A modelagem seguiu essas premissas e foi realizada em EMSO, um ambiente de simulação orientado a objetos. O modelo final foi baseado na proposta de modelagem em (MENDOZA et al., 2013), utilizando as mesmas hipóteses, e contém uma coluna de destilação com 197 pratos, um reboiler total, um tanque de distribuição, um compressor, um condensador auxiliar, três separadores de fluxo, dois misturadores de fluxo e três válvulas de expansão

\section{CONCLUSÕES}

De certo, está se construindo uma sólida base de conhecimento sobre as metodologias propostas na literatura que tem sido avaliadas e classificadas. Os resultados desta avaliação mostram que as metodologias de RTO atuais precisam maior desenvolvimento para se tornar uma ferramenta confiável para aplicações industriais. A redução da incerteza tanto nas medições quanto no modelo matemático são pontos importantes para conseguir melhorar as metodologias disponíveis. Atualmente em nosso grupo estão-se formulando e testando métodos que possam reduzir o nível de incerteza do RTO.

Esse projeto traz uma contribuição global frente aos softwares comerciais, que são na verdade um conjunto de softwares independentes integrados para formar o ciclo de RTO. As premissas para o desenvolvimento deste protótipo levam em consideração a capacidade de adaptação do fluxo de informação dentro do RTO, possibilitando a implementação de diversas metodologias disponíveis. Dentro desse contexto podemos considerar o protótipo como um laboratório para experimentação de novas metodologias em um cenário realista, pois pode ser implementado no processo real.

A criação desse protótipo é ganho para a engenharia química brasileira, já que está sendo desenvolvido utilizando ferramentas computacionais nacionais, assim como EMSO, e sem a dependência de softwares comercias. Dessa forma, o projeto representa uma continuação do desenvolvimento de softwares para engenharia química nacional, que começou com a criação da plataforma de modelagem, EMSO.

\section{REFERÊENCIAS}

BUNIN, G. A.; FRANÇOIS, G.; BONVIN, D. Performance of Real-Time Optimization Schemes - I. Sufficient Conditions for Feasibility and Optimality. 2013.

BUSCHMANN, F. et al. Pattern-Oriented Software Architecture. Chichester, UK: John Wiley \& Sons, 1996.

CAO, S.; RHINEHART, R. R. An efficient method for on-line identification of steady state. Journal of Process Control, v. 5, n. 6, p. 363-374, 1995.

DARBY, M. L. et al. RTO: An overview and assessment of current practice. J. Process Contr., v. 21, n. 6, p. 874, jul. 2011. 
FRIEDMAN, Y. Z. What's Wrong With Unit Closed Loop Optimization. Hydrocarb. process., v. 74, n. 10, p. 107-114, 1995.

GRACIANO, J. E. A.; MENDOZA, D. F.; LE ROUX, G. A. C. Assessing the Reliability of Different Real-Time Optimization Methodologies Using Monte Carlo Analysis 2013 AIChE Annual Meeting Online Proceedings. Anais...San Francisco: 2013

GRACIANO, J. E.; MENDOZA, D. F.; LE ROUX, G. A. C. Performance comparison of parameter estimation techniques for unidentifiable models. Computers \& Chemical Engineering, v. 64 , p. 24-40, maio 2014.

JOGWAR, S. S.; DAOUTIDIS, P. Dynamics and control of vapor recompression distillation. Journal of Process Control, v. 19, n. 10, p. 1737-1750, dez. 2009.

KORBEL, M. et al. Steady state identification for on-line data reconciliation based on wavelet transform and filtering. Computers \& Chemical Engineering, v. 63, p. 206-218, abr. 2014.

LE ROUX, G. A. C. et al. Improving steady-state identification. Proceedings of the 18th European Symposium on Computer Aided Process Engineering - ESCAPE 18, v. 25, p. 459-464, 2008.

LÜ, W. et al. A New Strategy of Integrated Control and On-line Optimization on High-purity Distillation Process. Chinese Journal of Chemical Engineering, v. 18, n. 1, p. 66-79, fev. 2010.

MARCHETTI, A.; CHACHUAT, B.; BONVIN, D. Modifier-Adaptation Methodology for RealTime Optimization. Industrial \& Engineering Chemistry Research, v. 48, n. 13, p. 6022-6033, jul. 2009.

MEIXELL, M. D.; GOCHENOUR, B.; CHEN, C.-C. Chapter 3 - Industrial Applications of Plant-Wide Equation-Oriented Process Modeling-2010. In: SUNDMACHE, K. (Ed.). Adv. Chem. Eng. Burlington: Elsevier, 2010. p. 152.

MENDOZA, D. F. et al. Real-Time Optimization of an Industrial-Scale Vapor Recompression Distillation Process. Model Validation and Analysis. Industrial \& Engineering Chemistry Research, v. 52, n. 16, p. 5735-5746, 24 abr. 2013.

ROBERTS, P. D. An algorithm for steady-state system optimization and parameter estimation. International Journal of Systems Science, v. 10, n. 7, p. 719-734, jul. 1979.

TAO, L. et al. Steady-state identification with gross errors for industrial process units. Proceedings of the 10th World Congress on Intelligent Control and Automation, n. 3, p. 41514154, jul. 2012. 